\title{
Somewhat Slightly Generalized Double Fuzzy Semicontinuous Functions
}

\author{
Fatimah M. Mohammed, ${ }^{1,2}$ M. S. M. Noorani, ${ }^{1}$ and A. Ghareeb ${ }^{3,4}$ \\ ${ }^{1}$ School of Mathematical Sciences, Universiti Kebangsaan Malaysia, 43600 UKM Bangi, Selangor, Malaysia \\ ${ }^{2}$ College of Education, Tikrit University, Iraq \\ ${ }^{3}$ Department of Mathematics, College of Science in Al-Zulfi, Majmaah University, Al-Zulfi, Saudi Arabia \\ ${ }^{4}$ Department of Mathematics, Faculty of Science, South Valley University, Qena, Egypt
}

Correspondence should be addressed to A. Ghareeb; nasserfuzt@hotmail.com

Received 18 April 2014; Revised 26 May 2014; Accepted 27 May 2014; Published 12 June 2014

Academic Editor: Nawab Hussain

Copyright (c) 2014 Fatimah M. Mohammed et al. This is an open access article distributed under the Creative Commons Attribution License, which permits unrestricted use, distribution, and reproduction in any medium, provided the original work is properly cited.

\begin{abstract}
The aim of this paper is to introduce the concepts of somewhat slightly generalized double fuzzy semicontinuous functions and somewhat slightly generalized double fuzzy semiopen functions in double fuzzy topological spaces. Some interesting properties and characterizations of these functions are introduced and discussed. Furthermore, the relationships among the new concepts are discussed with some necessary examples.
\end{abstract}

\section{Introduction}

In 1968, Chang [1] was the first to introduce the concept of fuzzy topological spaces. These spaces and their generalization are later developed by Goguen [2], who replaced the closed interval $[0,1]$ by more general lattice $L$. On the other hand, by the independent and parallel generalization of Kubiak and Šostak's [3, 4], made topology itself fuzzy besides their dependence on fuzzy set in 1985.

Various generalizations of the concept of fuzzy set have been done by many authors. In [5-10], Atanassove introduced the notion of intuitionistic fuzzy sets. Later Çoker [11] defined intuitionistic fuzzy topology in Chang's sense. Then, Mondal and Samanta [12] introduced the intuitionistic gradation of openness of fuzzy sets. Gutiérrez García and Rodabaugh [13], in 2005, replaced the term "intuitionistic" and concluded that the most appropriate work is under the name "double."

In 1980, Jain [14] introduced the notion of slightly continuous functions. On the other hand, Nour [15] defined slightly semicontinuous functions as a weak form of slight continuity and investigated their properties. In [16], Noiri introduced the concept of slightly $\beta$-continuous functions. Sudha et al. [17] introduced slightly fuzzy $\omega$-continuous functions. Also in 2004, Ekici and Caldas [18] introduced the notion of slight $\gamma$-continuity (slight $b$-continuity).
In this paper, the concepts of somewhat slightly generalized double fuzzy semicontinuous functions and somewhat slightly generalized double fuzzy semiopen functions are introduced. Several interesting properties and characterizations are introduced and discussed. Furthermore, the relationships among the concepts are obtained and established with some interesting counter examples.

\section{Preliminaries}

Throughout this paper, let $X$ be a nonempty set, $I$ the unit interval $[0,1], I_{0}=(0,1]$, and $I_{1}=[0,1)$. The family of all fuzzy sets in $X$ is denoted by $I^{X}$. $P_{t}(X)$ is the family of all fuzzy points in $X$. By $\underline{0}$ and $\underline{1}$ we denote the smallest and the greatest fuzzy sets on $X$. For a fuzzy set $\lambda \in I^{X}, \underline{1}-\lambda$ denotes its complement. Given a function $f: X \rightarrow Y, f(\lambda)$ and $f^{-1}(\lambda)$ defined the direct image and the inverse image of $f$, defined by $f(\lambda)(y)=\bigvee_{f(x)=y} \lambda(x)$ and $f^{-1}(\mu)(x)=\mu(f(x))$ for each $\lambda \in I^{X}, \mu \in I^{Y}$, and $x \in X$, respectively. All other notations are standard notations of fuzzy set theory.

Definition 1 (see $[12,13])$. A double fuzzy topology $\left(\tau, \tau^{*}\right)$ on $X$ is a pair of maps $\tau, \tau^{*}: I^{X} \rightarrow I$, which satisfies the following properties: 
(O1) $\tau(\lambda) \leq \underline{1}-\tau^{*}(\lambda)$ for each $\lambda \in I^{X}$;

(O2) $\tau\left(\lambda_{1} \wedge \lambda_{2}\right) \geq \tau\left(\lambda_{1}\right) \wedge \tau\left(\lambda_{2}\right)$ and $\tau^{*}\left(\lambda_{1} \wedge \lambda_{2}\right) \leq \tau^{*}\left(\lambda_{1}\right) \vee$ $\tau^{*}\left(\lambda_{2}\right)$ for each $\lambda_{1}, \lambda_{2} \in I^{X}$;

(O3) $\tau\left(\bigvee_{i \in \Gamma} \lambda_{i}\right) \geq \bigwedge_{i \in \Gamma} \tau\left(\lambda_{i}\right)$ and $\tau^{*}\left(\bigvee_{i \in \Gamma} \lambda_{i}\right) \leq \bigvee_{i \in \Gamma} \tau^{*}\left(\lambda_{i}\right)$ for each $\lambda_{i} \in I^{X}, i \in \Gamma$.

The triplet $\left(X, \tau, \tau^{*}\right)$ is called double fuzzy topological spaces (dfts, for short). A fuzzy set $\lambda$ is called an $(r, s)$-fuzzy open $\left((r, s)\right.$-fo, for short) if $\tau(\lambda) \geq r$ and $\tau^{*}(\lambda) \leq s, \lambda$ is called an $(r, s)$-fuzzy closed $((r, s)$-fc, for short) if and only if $\underline{1}-\lambda$ is an $(r, s)$-fo set, and $\lambda$ is called $(r, s)$-fuzzy clopen $((r, s)$ $\mathrm{fco}$, for short) if and only if $\lambda$ is $(r, s)$-fo set and $(r, s)$-fc set. Let $\left(X, \tau_{1}, \tau_{1}^{*}\right)$ and $\left(Y, \tau_{2}, \tau_{2}^{*}\right)$ be two dfts's. A function $f: X \rightarrow Y$ is said to be a double fuzzy continuous if and only if $\tau_{1}\left(f^{-1}(\nu)\right) \geq \tau_{2}(\nu)$ and $\tau_{1}^{*}\left(f^{-1}(\nu)\right) \leq \tau_{2}^{*}(\nu)$ for each $\nu \in I^{Y}$.

Before starting to present our results, there are two questions that we must ask ourselves. First, what is the difference between classical topology and double fuzzy topology? Secondly, where we can apply our results?

To answer the first question, we should know that double fuzzy sets and hence double fuzzy topological spaces deal with obscurities. In addition to that, we observed that the concept of double fuzzy topological spaces is a generalization of fuzzy topological spaces and classical topology. For example, when the first condition in Definition 1 does not hold, we get the definition of fuzzy topological spaces in KubiakŠostak's sense $[3,4]$. Also, in the same definition, when we replace $2^{X}$ with $I^{X}$, we will get results in double gradation fuzzifying topological spaces [19]. Appropriate changes can be made to get results in the classical topological spaces.

With regard to applications, since double fuzzy topology forms an extension of fuzzy topology and general topology, we think that our results can be applied in the fuzzy mathematics, which has many applications in different branches of engineering and ICT. For example, recently double fuzzy topological spaces have been applied to study sensor bias [20] and there exist well-established applications of fuzzy topological spaces in the areas of digital topology [21], image processing [22], and geographic information systems (GIS) problems [23].

Theorem 2 (see $[24,25])$. Let $\left(X, \tau, \tau^{*}\right)$ be a dfts. Then for each $r \in I_{0}, s \in I_{1}$, and $\lambda \in I^{X}$, one defines an operator $C_{\tau, \tau^{*}}$ : $I^{X} \times I_{0} \times I_{1} \rightarrow I^{X}$ as follows:

$$
\begin{gathered}
C_{\tau, \tau^{*}}(\lambda, r, s)=\bigwedge\left\{\mu \in I^{X} \mid \lambda \leq \mu, \tau(\underline{1}-\mu) \geq r,\right. \\
\left.\tau^{*}(\underline{1}-\mu) \leq s\right\} .
\end{gathered}
$$

For $\lambda, \mu \in I^{X}, r, r_{1}, r_{2} \in I_{0}$ and $s, s_{1}, s_{2} \in I_{1}$, the operator $C_{\tau, \tau^{*}}$ satisfies the following statements:
(C1) $C_{\tau, \tau^{*}}(\underline{0}, r, s)=\underline{0}$;
(C2) $\lambda \leq C_{\tau, \tau^{*}}(\lambda, r, s)$;
(C3) $C_{\tau, \tau^{*}}(\lambda, r, s) \vee C_{\tau, \tau^{*}}(\mu, r, s)=C_{\tau, \tau^{*}}(\lambda \vee \mu, r, s)$;

(C4) $C_{\tau, \tau^{*}}\left(\lambda, r_{1}, s_{1}\right) \leq C_{\tau, \tau^{*}}\left(\lambda, r_{2}, s_{2}\right)$ if $r_{1} \leq r_{2}$ and $s_{1} \geq s_{2}$;

(C5) $C_{\tau, \tau^{*}}\left(C_{\tau, \tau^{*}}(\lambda, r, s), r, s\right)=C_{\tau, \tau^{*}}(\lambda, r, s)$.

Theorem 3 (see $[24,25])$. Let $\left(X, \tau, \tau^{*}\right)$ be a dfts. Then for each $r \in I_{0}, s \in I_{1}$, and $\lambda \in I^{X}$, one defines an operator $I_{\tau, \tau^{*}}$ : $I^{X} \times I_{0} \times I_{1} \rightarrow I^{X}$ as follows:

$$
I_{\tau, \tau^{*}}(\lambda, r, s)=\bigvee\left\{\mu \in I^{X} \mid \mu \leq \lambda, \tau(\mu) \geq r, \tau^{*}(\mu) \leq s\right\} .
$$

For $\lambda, \mu \in I^{X}, r, r_{1}, r_{2} \in I_{0}$ and $s, s_{1}, s_{2} \in I_{1}$, the operator $I_{\tau, \tau^{*}}$ satisfies the following statements:

(I1) $I_{\tau, \tau^{*}}(\underline{1}-\lambda, r, s)=\underline{1}-C_{\tau, \tau^{*}}(\lambda, r, s)$;

(I2) $I_{\tau, \tau^{*}}(\underline{1}, r, s)=\underline{1}$;

(I3) $I_{\tau, \tau^{*}}(\lambda, r, s) \leq \lambda$;

(I4) $I_{\tau, \tau^{*}}(\lambda, r, s) \wedge I_{\tau, \tau^{*}}(\mu, r, s)=I_{\tau, \tau^{*}}(\lambda \wedge \mu, r, s)$;

(I5) $I_{\tau, \tau^{*}}\left(\lambda, r_{1}, s_{1}\right) \geq I_{\tau, \tau^{*}}\left(\lambda, r_{2}, s_{2}\right)$ if $r_{1} \leq r_{2}$ and $s_{1} \geq s_{2}$;

(I6) $I_{\tau, \tau^{*}}\left(I_{\tau, \tau^{*}}(\lambda, r, s), r, s\right)=I_{\tau, \tau^{*}}(\lambda, r, s)$;

(I7) If $I_{\tau, \tau^{*}}\left(C_{\tau, \tau^{*}}(\lambda, r, s), r, s\right)=\lambda$, then $C_{\tau, \tau^{*}}\left(I_{\tau, \tau^{*}}(\underline{1}-\right.$ $\lambda, r, s), r, s)=1-\lambda$.

Definition 4 (see [26]). Let $\left(X, \tau, \tau^{*}\right)$ be a dfts. For each $\lambda, \mu \in$ $I^{X}, r \in I_{0}$, and $s \in I_{1}$.

(1) A fuzzy set $\lambda$ is called $(r, s)$-fuzzy semiclosed (briefly, $(r, s)$-fsc $)$ if $I_{\tau, \tau^{*}}\left(C_{\tau, \tau^{*}}(\lambda, r, s), r, s\right) \leq \lambda . \lambda$ is called $(r, s)$-fuzzy semiopen (briefly, $(r, s)$-fso) if and only if $\underline{1}-\lambda$ is an $(r, s)$-fuzzy semiclosed set.

(2) An $(r, s)$-fuzzy semiclosure of $\lambda$ is defined by $\mathrm{SC}_{\tau, \tau^{*}}(\lambda, r, s)=\bigwedge\left\{\mu \in I^{X} \mid \lambda \leq \mu\right.$ and $\mu$ is $(r, s)$-fsc $\}$.

Definition 5 (see [26]). Let $\left(X, \tau, \tau^{*}\right)$ be a dfts. For each $\lambda, \mu \in$ $I^{X}, r \in I_{0}$ and $s \in I_{1}$.

(1) A fuzzy set $\lambda$ is called $(r, s)$-generalized fuzzy semiclosed (briefly, $(r, s)$-gfsc) if $\mathrm{SC}_{\tau, \tau^{*}}(\lambda, r, s) \leq \mu, \lambda \leq \mu$ and $\tau(\mu) \geq r, \tau^{*}(\mu) \leq s$. $\lambda$ is called $(r, s)$-generalized fuzzy semiopen (briefly, $(r, s)$-gfso) if and only if $\underline{1}-\lambda$ is $(r, s)$-gfsc set.

(2) An $(r, s)$-fuzzy generalized semiclosure of $\lambda$ is defined by $\operatorname{GSC}_{\tau, \tau^{*}}(\lambda, r, s)=\bigwedge\left\{\mu \in I^{X} \mid \lambda \leq \mu\right.$ and $\mu$ is $(r, s)$ gfsc\}.

(3) An $(r, s)$-fuzzy generalized semi-interior of $\lambda$ is defined by $\operatorname{GSI}_{\tau, \tau^{*}}(\lambda, r, s)=\bigvee\left\{\mu \in I^{X} \mid \mu \leq \lambda\right.$ and $\mu$ is $(r, s)$-gfso .

Definition 6 (see [27]). Let $\left(X, \tau_{1}, \tau_{1}^{*}\right)$ and $\left(Y, \tau_{2}, \tau_{2}^{*}\right)$ be dfts's. A function $f:\left(X, \tau_{1}, \tau_{1}^{*}\right) \rightarrow\left(Y, \tau_{2}, \tau_{2}^{*}\right)$ is called

(1) slightly double fuzzy continuous (briefly, sdfc) if for every $\lambda \in I^{X}, \mu \in I^{Y}, r \in I_{0}$, and $s \in I_{1}$ such that $\mu$ is $(r, s)$-fco set and $f(\lambda) \leq \mu$, there exists $\nu \in I^{X}$ such that $\tau_{1}(\nu) \geq r, \tau_{1}^{*}(\nu) \leq s, \lambda \leq \nu$, and

$$
f(\nu) \leq \mu,
$$


(2) slightly generalized double fuzzy semicontinuous (briefly, sgdfsc) if for each $\lambda \in I^{X}, \mu \in I^{Y}, r \in I_{0}$, and $s \in I_{1}$ such that $\mu$ is $(r, s)$-fco set and $f(\lambda) \leq \mu$, there exists an $(r, s)$-gfso set $v \in I^{X}$ such that $\lambda \leq v$ and

$$
f(\nu) \leq \mu
$$

\section{Somewhat Slightly Generalized Double Fuzzy Semicontinuous Functions}

Definition 7. Let $\left(X, \tau_{1}, \tau_{1}^{*}\right)$ and $\left(Y, \tau_{2}, \tau_{2}^{*}\right)$ be dfts's. A function $f:\left(X, \tau_{1}, \tau_{1}^{*}\right) \rightarrow\left(Y, \tau_{2}, \tau_{2}^{*}\right)$ is called somewhat slightly generalized double fuzzy semicontinuous (briefly, swsgdfsc) if for each fuzzy set $\lambda \in I^{X}, \mu \in I^{Y}, r \in I_{0}$, and $s \in I_{1}$ such that $f^{-1}(\mu) \neq \underline{0}$ and $f(\lambda) \leq \mu$, there exists an $(r, s)$-gfso set $\underline{0} \neq v \in I^{X}$ such that $\lambda \leq v$ and

$$
\nu \leq f^{-1}(\mu) .
$$

Definition 8. A fuzzy set $\lambda$ in a $\mathrm{dfts}\left(X, \tau, \tau^{*}\right)$ is called $(r, s)$ generalized fuzzy semidense (resp., $(r, s)$-fuzzy-dense $e^{*}$ ) set if there exists no $(r, s)$-gfsc (resp., $(r, s)$-fco) set $\mu \in I^{X}, r \in I_{0}$, and $s \in I_{1}$ such that

$$
\lambda<\mu<\underline{1} .
$$

Example 9. (1) Let $X=\{a, b\}$. Define $\lambda_{1}$ and $\lambda_{2}$ as follows:

$$
\begin{array}{ll}
\lambda_{1}(a)=0.1, & \lambda_{1}(b)=0.2, \\
\lambda_{2}(a)=0.8, & \lambda_{2}(b)=0.7 .
\end{array}
$$

And define $\tau(\lambda)$ and $\tau^{*}(\lambda)$ as follows:

$$
\begin{gathered}
\tau(\lambda)= \begin{cases}1, & \text { if } \lambda=\underline{0} \text { or } \underline{1} ; \\
\frac{1}{3}, & \text { if } \lambda=\lambda_{1} ; \\
\frac{2}{3}, & \text { if } \lambda=\lambda_{2} ; \\
0, & \text { otherwise; }\end{cases} \\
\tau^{*}(\lambda)= \begin{cases}0, & \text { if } \lambda=\underline{0} \text { or } \underline{1} ; \\
\frac{2}{3}, & \text { if } \lambda=\lambda_{1} ; \\
\frac{1}{3}, & \text { if } \lambda=\lambda_{2} ; \\
1, & \text { otherwise. }\end{cases}
\end{gathered}
$$

So, if $\lambda(a)=0.9, \lambda(b)=0.8$, then there exists no $(1 / 3,2 / 3)$ gfsc set $\mu$ in $I^{X}$ such that $\lambda<\mu<\underline{1}$. Therefore, $\lambda$ is $(1 / 3,2 / 3)$ generalized fuzzy semidense set in $I^{X}$.

(2) In (1), let $\lambda_{1}$ and $\lambda_{2}$ be defined as follows:

$$
\begin{array}{ll}
\lambda_{1}(a)=0.1, & \lambda_{2}(b)=0.2, \\
\lambda_{2}(a)=0.9, & \lambda_{2}(b)=0.8 .
\end{array}
$$

So, if $\lambda(a)=0.8, \lambda(b)=0.9$, then there exists no $(1 / 3,2 / 3)$ fco set $\mu$ in $I^{X}$ such that $\lambda<\mu<1$. Therefore, $\lambda$ is $(1 / 3,2 / 3)$ fuzzy-dense $e^{*}$ set in $I^{X}$.
Definition 10. Let $\left(X, \tau, \tau^{*}\right)$ be a dfts. For a fuzzy set $\lambda \in I^{X}$, $r \in I_{0}$, and $s \in I_{1}, I_{\tau, \tau^{*}}^{*}$ and $C_{\tau, \tau^{*}}^{*}$ are defined as follows:

(1) $I_{\tau, \tau^{*}}^{*}(\lambda, r, s)=\bigvee\left\{\mu \in I^{X} \mid \mu \leq \lambda\right.$ and $\mu$ is $(r, s)$-fco $\}$;

(2) $C_{\tau, \tau^{*}}^{*}(\lambda, r, s)=\bigwedge\left\{\mu \in I^{X} \mid \lambda \leq \mu\right.$ and $\mu$ is $(r, s)$-fco $\}$.

Proposition 11. Let $\left(X, \tau_{1}, \tau_{1}^{*}\right)$ and $\left(Y, \tau_{2}, \tau_{2}^{*}\right)$ be dfts's, and let $f:\left(X, \tau_{1}, \tau_{1}^{*}\right) \rightarrow\left(Y, \tau_{2}, \tau_{2}^{*}\right)$ be any function. Then the following are equivalent.

(1) $f$ is swsgdfsc function.

(2) If $\lambda$ is an $(r, s)$-fco set such that $f^{-1}(\lambda) \neq \underline{1}$ and $\lambda \leq$ $f(\underline{1}-\nu)$, for each $\nu \in I^{X}, r \in I_{0}$, and $s \in I_{1}$, then there exists an $(r, s)$-gfsc set $\mu \leq \underline{1}-v \in I^{X}$ such that $\mu \geq$ $f^{-1}(\lambda)$.

(3) If $\lambda$ is $(r, s)$-gfs-dense set in $I^{X}$, then $f(\lambda)$ is $(r, s)$ fuzzy-dense ${ }^{*}$ set in $I^{Y}$ such that every $(r, s)$-fco set $\mu \leq f(\underline{1}-\nu)$, for each $\nu \in I^{X}, r \in I_{0}$, and $s \in I_{1}$.

Proof. (1) $\Rightarrow$ (2) Suppose $f$ is swsgdfsc function, and let $\lambda$ be any $(r, s)$-fco set in $I^{Y}$ such that $f^{-1}(\lambda) \neq \underline{1}$ and $\lambda \leq f(\underline{1}-\nu)$, for each $v \in I^{X}, r \in I_{0}$, and $s \in I_{1}$. Then, $\underline{1}-\lambda$ is $(r, s)$-fco in $I^{Y}$ such that $f^{-1}(\underline{1}-\lambda) \neq \underline{0}$ and $f(\nu) \leq \underline{1}-\lambda$. Then by the hypothesis, there exists an $(r, s)$-gfso set $\underline{0} \neq \alpha \in I^{X}, r \in I_{0}$, and $s \in I_{1}$ such that $\nu \leq \alpha$ and $\alpha \leq f^{-1}(\underline{1}-\lambda)$. That is, $\underline{1}-\alpha$ is an $(r, s)$-gfsc set and

$$
\underline{1}-\alpha \geq \underline{1}-f^{-1}(\underline{1}-\lambda)=f^{-1}(\lambda) .
$$

Put $\underline{1}-\alpha=\mu$. Then $\mu$ is an $(r, s)$-gfsc set in $I^{X}$ such that $\mu \geq \bar{f}^{-1}(\lambda)$.

(2) $\Rightarrow$ (3) Let $\lambda$ be an $(r, s)$-gfs-dense set in $I^{X}$, and suppose that $f(\lambda)$ is not a fuzzy-dense ${ }^{*}$ set in $I^{Y}$, such that each $(r, s)$-fco set $\mu \leq f(\underline{1}-\nu)$, for each $\nu \in I^{X}, r \in I_{0}$, and $s \in I_{1}$. Then, there exists an $(r, s)$-fco set $\alpha \in I^{Y}$ such that

$$
f(\lambda)<\alpha<\underline{1},
$$

since

$$
\alpha<\underline{1}, \quad f^{-1}(\alpha) \neq \underline{1} .
$$

Now, $\alpha$ is an $(r, s)$-fco set such that $f^{-1}(\alpha) \neq \underline{1}$ and $f(\underline{1}-$ $v) \geq \alpha$, for each $v \in I^{X}, r \in I_{0}$, and $s \in I_{1}$. Then by the hypothesis, there exists an $(r, s)$-gfsc set $\gamma \leq \underline{1}-\nu \in I^{X}$ such that $\gamma \geq f^{-1}(\alpha)$.

But

$$
f^{-1}(\alpha)>f^{-1}(f(\lambda))=\lambda .
$$

That is, $\gamma \geq \lambda$. Therefore, there exists an $(r, s)$-gfsc set $\gamma \in$ $I^{X}, r \in I_{0}$, and $s \in I_{1}$ such that $\gamma \geq \lambda$, which is a contradiction. Therefore, $f(\lambda)$ is an $(r, s)$-fuzzy dense $e^{*}$ set in $I^{Y}$ such that

$$
\gamma \leq f(\underline{1}-\nu),
$$

for each $\nu \in I^{X}$ and $(r, s)$-fco set $\gamma \in I^{Y}$. 
(3) $\Rightarrow$ (1) Let $\lambda$ be an $(r, s)$-fco set such that $f^{-1}(\lambda) \neq \underline{0}$ and $f(\nu) \leq \lambda$, for each $v \in I^{X}, r \in I_{0}$, and $s \in I_{1}$. Then, $\lambda \neq \underline{0}$. Now, suppose that $\nu \leq \alpha$ and $\operatorname{GSI}_{\tau, \tau^{*}}\left(f^{-1}(\lambda), r, s\right)=\underline{0} \in I^{\bar{X}}$. Then,

$$
\operatorname{GSC}_{\tau, \tau^{*}}\left(\underline{1}-f^{-1}(\lambda), r, s\right)=\underline{1} \in I^{X} .
$$

That is, $\underline{1}-f^{-1}(\lambda)$ is an $(r, s)$-gfs-dense in $I^{X}$. Then by (3), $f\left(\underline{1}-f^{-1}(\lambda)\right)$ is an $(r, s)$-fuzzy dense ${ }^{*}$ set such that there exists an $(r, s)$-fco set $\mu \leq f(\underline{1}-\nu)$, for each $\nu \in I^{X}, r \in I_{0}$, and $s \in I_{1}$.

But

$$
f\left(\underline{1}-f^{-1}(\lambda)\right)=f\left(f^{-1}(\underline{1}-\lambda)\right) \leq \underline{1}-\lambda<\underline{1},
$$

since $\underline{1}-\lambda$ is an $(r, s)$-fco and

$$
\begin{gathered}
f\left(\underline{1}-f^{-1}(\lambda)\right) \leq \underline{1}-\lambda, \\
C_{\tau, \tau^{*}}^{*}\left(f\left(\underline{1}-f^{-1}(\lambda)\right), r, s\right) \leq \underline{1}-\lambda .
\end{gathered}
$$

That is,

$$
\underline{1}-\lambda \geq \underline{1} \Longrightarrow \lambda=\underline{0},
$$

which is a contradiction, since $\lambda \neq \underline{0}$. Therefore, $v \leq \alpha$ and $\operatorname{GSI}_{\tau, \tau^{*}}\left(f^{-1}(\lambda), r, s\right) \neq \underline{0}$. So $f$ is swsgdfsc.

\section{Somewhat Slightly Generalized Double Fuzzy Semiopen Functions}

Definition 12. Let $\left(X, \tau_{1}, \tau_{1}^{*}\right)$ and $\left(Y, \tau_{2}, \tau_{2}^{*}\right)$ be dfts's. A function $f:\left(X, \tau_{1}, \tau_{1}^{*}\right) \rightarrow\left(Y, \tau_{2}, \tau_{2}^{*}\right)$ is called

(1) generalized double fuzzy semiopen (briefly, gdfso) if for each $(r, s)$-gfso set $\lambda \in I^{X}, r \in I_{0}$, and $s \in I_{1}, f(\lambda)$ is an $(r, s)$-gfso in $I^{Y}$;

(2) slightly generalized double fuzzy semiopen (briefly, sgdfso) if for each $(r, s)$-gfso set $\lambda \in I^{X}$ and each $\mu \in I^{X}, r \in I_{0}$, and $s \in I_{1}$ such that $\lambda \leq \mu, f(\lambda)$ is an $(r, s)$-fco set in $I^{Y}$ and

$$
f(\lambda) \leq f(\mu) ;
$$

(3) somewhat generalized double fuzzy semiopen (briefly, swgdfso) if for each $(r, s)$-gfso set $\underline{0} \neq \lambda \in I^{X}$, $r \in I_{0}$, and $s \in I_{1}$, there exists an $(r, s)$-gfso set $\underline{0} \neq \mu \in I^{Y}$ such that

$$
f(\lambda) \geq \mu
$$

(4) somewhat slightly generalized double fuzzy semiopen (briefly, swsgdfso) if for each $(r, s)$-gfso set $\underline{0} \neq \lambda \in I^{X}$ such that $\lambda \leq \nu$ and for each $v \in I^{X}, r \in I_{0}$, and $s \in I_{1}$, there exists an $(r, s)$-fco set $\underline{0} \neq \mu \in I^{Y}, \mu \leq f(\nu)$ such that

$$
f(\lambda) \geq \mu \text {. }
$$

That is, $I_{\tau, \tau^{*}}^{*}(f(\lambda), r, s) \neq \underline{0}$, and there exists an $(r, s)$ fco set $\mu$ such that $f(\nu) \geq \mu$ and $\lambda \leq \nu$, for each $\nu \in I^{X}$, $r \in I_{0}$, and $s \in I_{1}$.

Proposition 13. Let $\left(X, \tau_{1}, \tau_{1}^{*}\right),\left(Y, \tau_{2}, \tau_{2}^{*}\right)$, and $\left(Z, \tau_{3}, \tau_{3}^{*}\right)$ be dfts's. If $f:\left(X, \tau_{1}, \tau_{1}^{*}\right) \rightarrow\left(Y, \tau_{2}, \tau_{2}^{*}\right)$ and $g:\left(Y, \tau_{2}, \tau_{2}^{*}\right) \rightarrow$ $\left(Z, \tau_{3}, \tau_{3}^{*}\right)$ are swsgdfso functions, then $g \circ f:\left(X, \tau_{1}, \tau_{1}^{*}\right) \rightarrow$ $\left(Z, \tau_{3}, \tau_{3}^{*}\right)$ is a swsgdfso function.

Proof. Let $\underline{0} \neq \lambda \in I^{X}$ be an $(r, s)$-gfso set $r \in I_{0}$ and $s \in I_{1}$ such that $\lambda \leq \mu$, for each fuzzy set $\mu \in I^{X}, r \in I_{0}$, and $s \in I_{1}$. Since $f$ is swsgdfso, then there exists an $(r, s)$-fco set $\underline{0} \neq \nu \in$ $I^{Y}$, and $f(\mu) \geq v$ such that $f(\lambda) \geq v$.

Now, $\left.\operatorname{GSI}_{\tau, \tau^{*}}(f(\lambda), r, s)\right)$ is an $(r, s)$-gfso in $I^{Y}$ such that

$$
\begin{gathered}
\operatorname{GSI}_{\tau, \tau^{*}}(f(\lambda), r, s) \neq \underline{0}, \\
\operatorname{GSI}_{\tau, \tau^{*}}(f(\lambda), r, s) \leq f(\mu),
\end{gathered}
$$

for each $f(\mu) \in I^{Y}$.

Since $g$ is swsgdfso, then there exists an $(r, s)$-fco set $\underline{0} \neq \gamma \in I^{Z}$ and $\gamma \leq g(f(\mu))$ such that

$$
\gamma \leq g\left(\operatorname{GSI}_{\tau, \tau^{*}}(f(\lambda), r, s)\right) .
$$

But

$$
g\left(\operatorname{GSI}_{\tau, \tau^{*}}(f(\lambda), r, s)\right) \leq g(f(\lambda)) .
$$

Thus, there exists an $(r, s)$-fco set $\underline{0} \neq \gamma \in I^{Z}$ and

$$
(g \circ f)(\mu) \geq \gamma,
$$

such that

$$
(g \circ f)(\lambda) \geq \gamma .
$$

Therefore, $g \circ f$ is swsgdfso.

Proposition 14. Let $\left(X, \tau_{1}, \tau_{1}^{*}\right)$ and $\left(Y, \tau_{2}, \tau_{2}^{*}\right)$ be dfts's, and let $f:\left(X, \tau_{1}, \tau_{1}^{*}\right) \rightarrow\left(Y, \tau_{2}, \tau_{2}^{*}\right)$ be a bijective function. Then the following are equivalent.

(1) $f$ is swsgdfso function.

(2) If $\lambda$ is an $(r, s)$-gfsc set in $I^{X}$ such that $f(\lambda) \neq \underline{1}$ and $\lambda \geq \nu$ for each $\nu \in I^{X}$, then there exists an $(r, s)$ foo set $\mu \in I^{Y}, \mu \neq \underline{1}$, and $f(\nu) \leq \mu$ such that $f(\lambda) \leq$ $\mu$.

Proof. (1) $\Rightarrow$ (2) Let $\lambda$ be an $(r, s)$-gfsc set in $I^{X}$ such that $f(\lambda) \neq \underline{1}$ and $\lambda \geq \nu$, for each $\nu \in I^{X}, r \in I_{0}$, and $s \in I_{1}$. Then, $\underline{1}-\lambda$ is an $(r, s)$-gfso set in $I^{X}$ such that $f(\underline{1}-\lambda) \neq \underline{0}$ and $\underline{1}-\lambda \leq \underline{1}-v$, for each $v \in I^{X}$. So

$$
\underline{1}-\lambda \neq \underline{0} .
$$

Since $f$ is a swsgdfso, then there exists an $(r, s)$-fco set $\underline{0} \neq \delta \in$ $I^{Y}$ and $f(\underline{1}-\nu) \geq \delta$ such that

$$
f(\underline{1}-\lambda) \geq \delta .
$$




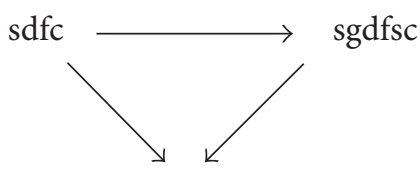

swsgdfsc

gdfso $\longrightarrow$ swgdfso

(a)

sgdfso

(b)

(c)

FIgURE 1

Now, $\underline{1}-\delta$ is an $(r, s)$-fco set in $I^{Y}$ such that $\underline{1}-\delta \neq \underline{1}$ and $\underline{1}-\delta \geq f(\nu)$ such that

$$
\underline{1}-\delta \geq f(\lambda)
$$

Take

$$
\underline{1}-\delta=\mu
$$

so (2) is proved.

(2) $\Rightarrow$ (1) Let $\lambda \neq \underline{0}$ be any $(r, s)$-gfso set in $I^{X}$ such that $\lambda \leq \nu$, for each $\nu \in I^{X}$. Then, $\underline{1}-\lambda$ is an $(r, s)$-gfsc set in $I^{X}$ such that $\underline{1}-\lambda \neq \underline{1}$ and $\underline{1}-\lambda \geq \underline{1}-v$ for each $\nu \in I^{X} r \in I_{0}$ and $s \in I_{1}$. Now,

$$
f(\underline{1}-\lambda)=\underline{1}-f(\lambda) \neq \underline{1} .
$$

For, if $\underline{1}-f(\lambda)=\underline{1}$, then

$$
f(\lambda)=\underline{0} \Longrightarrow \lambda=\underline{0} .
$$

Hence by the hypothesis, there exists an $(r, s)$-fco set $\mu \in I^{Y}$, $\underline{1} \neq \mu \geq f(\underline{1}-\nu)$, such that

$$
f(\underline{1}-\lambda) \leq \mu \text {. }
$$

That is,

$$
\underline{0} \neq \underline{1}-\mu \leq f(\nu)
$$

such that

$$
\underline{1}-\mu \leq f(\lambda) \text {. }
$$

Let $\underline{1}-\mu=\gamma$. Then, $\gamma \neq \underline{0}$ is an $(r, s)$-fco set in $I^{Y}$ such that $f(\nu) \geq \gamma$ and $f(\lambda) \geq \gamma$. Therefore, $f$ is swsgdfso function.

\section{Interrelations}

The following implication illustrates the relationships between different functions in Figure 1.

None of these implications is reversible where $A \rightarrow B$ represents $A$ implies $B$, as shown by the following examples.

Example 15. Let $X=\{a, b\}$.

(1) Let $f:\left(X, \tau_{1}, \tau_{1}^{*}\right) \rightarrow\left(X, \tau_{2}, \tau_{2}^{*}\right)$ be the identity function. Define $\lambda_{1}, \lambda_{2}, \mu_{1}$, and $\mu_{2}$ as follows:

$$
\begin{array}{ll}
\lambda_{1}(a)=0.1, & \lambda_{1}(b)=0.2, \\
\lambda_{2}(a)=0.9, & \lambda_{2}(b)=0.8, \\
\mu_{1}(a)=0.5, & \mu_{1}(b)=0.5, \\
\mu_{2}(a)=1.0, & \mu_{2}(b)=0.7 .
\end{array}
$$

And define $\left(\tau_{1}, \tau_{1}^{*}\right)$ and $\left(\tau_{2}, \tau_{2}^{*}\right)$ as follows:

$$
\tau_{1}(\lambda)= \begin{cases}1, & \text { if } \lambda \in\{\underline{0}, \underline{1}\} \\ \frac{1}{4}, & \text { if } \lambda=\lambda_{1}, \\ \frac{1}{8}, & \text { if } \lambda=\lambda_{2}, \\ 0, & \text { otherwise }\end{cases}
$$

$$
\tau_{1}^{*}(\lambda)= \begin{cases}0, & \text { if } \lambda \in\{\underline{0}, \underline{1}\} \\ \frac{1}{8}, & \text { if } \lambda=\lambda_{1}, \\ \frac{1}{4}, & \text { if } \lambda=\lambda_{2} \\ 1, & \text { otherwise }\end{cases}
$$

$$
\tau_{2}(\lambda)= \begin{cases}1, & \text { if } \lambda \in\{\underline{0}, \underline{1}\}, \\ \frac{1}{4}, & \text { if } \lambda=\mu_{1}, \\ \frac{1}{8}, & \text { if } \lambda=\mu_{2}, \\ 0, & \text { otherwise, }\end{cases}
$$

Then, $f$ is sgdfsc function but not sdfc.

(2) In (1), $f$ is swsgdfsc function but not sdfc.

(3) Let $f:\left(X, \tau_{1}, \tau_{1}^{*}\right) \rightarrow\left(X, \tau_{2}, \tau_{2}^{*}\right)$ be a function defined by

$$
f(a)=a, \quad f(b)=a .
$$

Define $\lambda_{1}, \mu_{1}$, and $\mu_{2}$ as follows:

$$
\begin{array}{ll}
\lambda_{1}(a)=1.0, & \lambda_{1}(b)=0.9, \\
\mu_{1}(a)=0.0, & \mu_{1}(b)=0.2, \\
\mu_{2}(a)=1.0, & \mu_{2}(b)=0.8,
\end{array}
$$


and define $\left(\tau_{1}, \tau_{1}^{*}\right)$ and $\left(\tau_{2}, \tau_{2}^{*}\right)$ as follows:

$$
\begin{gathered}
\tau_{1}(\lambda)= \begin{cases}1, & \text { if } \lambda \in\{\underline{0}, \underline{1}\}, \\
\frac{1}{2}, & \text { if } \lambda=\lambda_{1}, \\
0, & \text { otherwise, }\end{cases} \\
\tau_{1}^{*}(\lambda)= \begin{cases}0, & \text { if } \lambda \in\{\underline{0}, \underline{1}\}, \\
\frac{1}{2}, & \text { if } \lambda=\lambda_{1}, \\
1, & \text { otherwise, }\end{cases} \\
\tau_{2}(\lambda)= \begin{cases}1, & \text { if } \lambda \in\{\underline{0}, \underline{1}\}, \\
\frac{1}{3}, & \text { if } \lambda=\mu_{1}, \\
\frac{2}{3}, & \text { if } \lambda=\mu_{2}, \\
0, & \text { otherwise, }\end{cases} \\
\tau_{2}^{*}(\lambda)= \begin{cases}0, & \text { if } \lambda \in\{\underline{0}, \underline{1}\}, \\
\frac{2}{3}, & \text { if } \lambda=\mu_{1}, \\
\frac{1}{3}, & \text { if } \lambda=\mu_{2}, \\
1, & \text { otherwise. }\end{cases}
\end{gathered}
$$

Then, $f$ is swsgdfsc function but not sgdfsc.

(4) Let $f:\left(X, \tau_{1}, \tau_{1}^{*}\right) \rightarrow\left(X, \tau_{2}, \tau_{2}^{*}\right)$ be the identity function. Define $\lambda_{1}, \lambda_{2}$, and $\mu$ as follows:

$$
\begin{array}{ll}
\lambda_{1}(a)=0.0, & \lambda_{1}(b)=1.0, \\
\lambda_{2}(a)=1.0, & \lambda_{2}(b)=0.0, \\
\mu(a)=0.0, & \mu(b)=0.3 .
\end{array}
$$

And define $\left(\tau_{1}, \tau_{1}^{*}\right)$ and $\left(\tau_{2}, \tau_{2}^{*}\right)$ as follows:

$$
\begin{gathered}
\tau_{1}(\lambda)= \begin{cases}1, & \text { if } \lambda \in\{\underline{0}, \underline{1}\}, \\
\frac{1}{3}, & \text { if } \lambda=\lambda_{1}, \\
\frac{2}{3}, & \text { if } \lambda=\lambda_{2}, \\
0, & \text { otherwise, }\end{cases} \\
\tau_{1}^{*}(\lambda)= \begin{cases}0, & \text { if } \lambda \in\{\underline{0}, \underline{1}\}, \\
\frac{2}{3}, & \text { if } \lambda=\lambda_{1}, \\
\frac{1}{3}, & \text { if } \lambda=\lambda_{2}, \\
1, & \text { otherwise, }\end{cases}
\end{gathered}
$$

$$
\begin{gathered}
\tau_{2}(\lambda)= \begin{cases}1, & \text { if } \lambda \in\{\underline{0}, \underline{1}\}, \\
\frac{1}{3}, & \text { if } \lambda=\mu, \\
0, & \text { otherwise, }\end{cases} \\
\tau_{2}^{*}(\lambda)= \begin{cases}0, & \text { if } \lambda \in\{\underline{0}, \underline{1}\} \\
\frac{2}{3}, & \text { if } \lambda=\mu, \\
1, & \text { otherwise. }\end{cases}
\end{gathered}
$$

Then, $f$ is swgdfso function but not gdfso.

(5) Let $f:\left(X, \tau_{1}, \tau_{1}^{*}\right) \rightarrow\left(X, \tau_{2}, \tau_{2}^{*}\right)$ be the identity function. Define $\lambda_{1}, \mu_{1}$, and $\mu_{2}$ as follows:

$$
\begin{array}{cc}
\lambda_{1}(a)=0.1, & \lambda_{1}(b)=0.1, \\
\mu_{1}(a)=0.05, & \mu_{1}(b)=0.02, \\
\mu_{2}(a)=0.95, & \mu_{2}(b)=0.98 .
\end{array}
$$

And define $\left(\tau_{1}, \tau_{1}^{*}\right)$ and $\left(\tau_{2}, \tau_{2}^{*}\right)$ as follows:

$$
\tau_{1}(\lambda)= \begin{cases}1, & \text { if } \lambda \in\{\underline{0}, \underline{1}\} \\ \frac{1}{3}, & \text { if } \lambda=\lambda_{1}, \\ 0, & \text { otherwise, }\end{cases}
$$

$$
\tau_{1}^{*}(\lambda)= \begin{cases}0, & \text { if } \lambda \in\{\underline{0}, \underline{1}\}, \\ \frac{2}{3}, & \text { if } \lambda=\lambda_{1}, \\ 1, & \text { otherwise, }\end{cases}
$$$$
\tau_{2}(\lambda)= \begin{cases}1, & \text { if } \lambda \in\{\underline{0}, \underline{1}\}, \\ \frac{1}{3}, & \text { if } \lambda=\mu_{1}, \\ \frac{2}{3}, & \text { if } \lambda=\mu_{2}, \\ 0, & \text { otherwise, }\end{cases}
$$

Then, $f$ is swsgdfso function but not sgdfso.

\section{Conflict of Interests}

The authors declare that there is no conflict of interests regarding this paper.

\section{Acknowledgments}

The authors would like to acknowledge the financial support received from Universiti Kebangsaan Malaysia under the 
research Grant GUP-2013-040. The authors also wish to gratefully acknowledge all those who have generously given their time to referee their paper.

\section{References}

[1] C. L. Chang, "Fuzzy topological spaces," Journal of Mathematical Analysis and Applications, vol. 24, no. 1, pp. 182-190, 1968.

[2] J. A. Goguen, "L-fuzzy sets," Journal of Mathematical Analysis and Applications, vol. 18, no. 1, pp. 145-174, 1967.

[3] T. Kubiak, On fuzzy topologies [Ph.D. thesis], A. Mickiewicz, Poznan, Poland, 1985.

[4] A. P. Šostak, "On a fuzzy topological structure," in Proceedings of the 13th Winter School on Abstract Analysis. Section of Topology, pp. 89-103, Circolo Matematico di Palermo, Palermo, Italy, 1985.

[5] K. Atanassov and S. Stoeva, "Intuitionistic fuzzy sets," in Proceedings of the Polish Symposium on Interval and Fuzzy Mathematics, pp. 23-26, Poznan, Poland, August 1983.

[6] K. T. Atanassov, "Intuitionistic fuzzy sets," Fuzzy Sets and Systems, vol. 20, no. 1, pp. 87-96, 1986.

[7] K. T. Atanassov, "New operations defined over the intuitionistic fuzzy sets," Fuzzy Sets and Systems, vol. 61, no. 2, pp. 137-142, 1994.

[8] K. T. Atanassov, "More on intuitionistic fuzzy sets," Fuzzy Sets and Systems, vol. 33, no. 1, pp. 37-45, 1989.

[9] K. T. Atanassov, "Remarks on the intuitionistic fuzzy sets-III," Fuzzy Sets and Systems, vol. 75, no. 3, pp. 401-402, 1995.

[10] K. Atanassov, Intuitionistic Fuzzy Sets, Physica, New York, NY, USA, 1999.

[11] D. Çoker, "An introduction to intuitionistic fuzzy topological spaces," Fuzzy Sets and Systems, vol. 88, no. 1, pp. 81-89, 1997.

[12] T. K. Mondal and S. K. Samanta, "On intuitionistic gradation of openness," Fuzzy Sets and Systems, vol. 131, no. 3, pp. 323-336, 2002.

[13] J. Gutiérrez García and S. E. Rodabaugh, "Order-theoretic, topological, categorical redundancies of interval-valued sets, grey sets, vague sets, interval-valued "intuitionistic" sets, "intuitionistic" fuzzy sets and topologies," Fuzzy Sets and Systems, vol. 156, no. 3, pp. 445-484, 2005.

[14] R. C. Jain, The role of regularly open sets in general topology [Ph.D. thesis], Meerut University, Institute of Advenced Studied, Meerut-India, 1990.

[15] T. M. Nour, "Slightly semi-continuous functions," Bulletin of Calcutta Mathematical Society, vol. 87, pp. 187-195, 1995.

[16] T. Noiri, "Slightly $\beta$-continuous functions," International Journal of Mathematics and Mathematical Sciences, vol. 28, no. 8, pp. 469-478, 2001.

[17] M. Sudha, E. Roja, and M. K. Uma, "Slightly fuzzy $\omega$-continuous mappings," International Journal of Mathematical Analysis, vol. 5, no. 13-16, pp. 779-787, 2011.

[18] E. Ekici and M. Caldas, "Slightly $\gamma$-continuous functions," Boletim da Sociedade Paranaense de Matematica, vol. 22, no. 2, pp. 63-74, 2004.

[19] R. N. Bhaumik and S. E. Abbas, "(L, M)-intuitionistic fuzzy topological spaces," Bulletin of Kerala Mathematics Association, vol. 5, pp. 25-47, 2008.

[20] K. Dong, H. Wang, and Y. Liu, "Formation track association algorithm in the presence of sensor bias based on double fuzzy topology," Applied Mechanics and Materials, vol. 536-537, pp. 329-332, 2014.
[21] P. K. Saha, "Fuzzy digital topology and geometry and their applications to medical imaging," in Pattern Recognition and Machine Intelligence, vol. 8251, pp. 13-29, Springer, Berlin, Germany, 2013.

[22] I. Bloch, "On fuzzy distances and their use in image processing under imprecision," Pattern Recognition, vol. 32, no. 11, pp. 18731895, 1999.

[23] K. Liu and W. Shi, "Quantitative fuzzy topological relations of spatial objects by induced fuzzy topology," International Journal of Applied Earth Observation and Geoinformation, vol. 11, no. 1, pp. 38-45, 2009.

[24] M. Demirci and D. Çoker, "An introduction to intuitionistic fuzzy topological spaces in Šostak's sense," Busefal, vol. 67, pp. 67-76, 1996.

[25] Y. C. Kim and S. E. Abbas, "Several types of fuzzy regular spaces," Indian Journal of Pure and Applied Mathematics, vol. 35, no. 4, pp. 481-500, 2004.

[26] S. E. Abbas, "Several types of double fuzzy semiclosed sets," The Journal of Fuzzy Mathematics, vol. 20, pp. 89-102, 2012.

[27] F. M. Mohammed, M. S. M. Noorani, and A. Ghareeb, "Slightly double fuzzy continuous functions," Journal of the Egyptian Mathematical Society, 2014. 


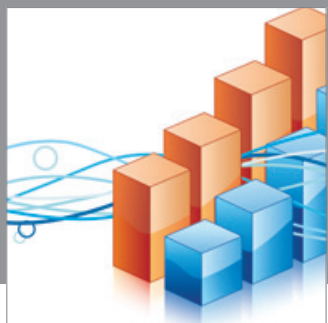

Advances in

Operations Research

mansans

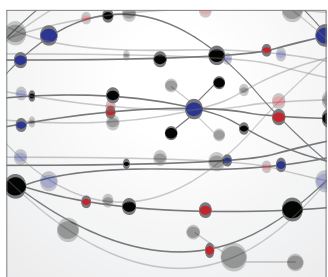

The Scientific World Journal
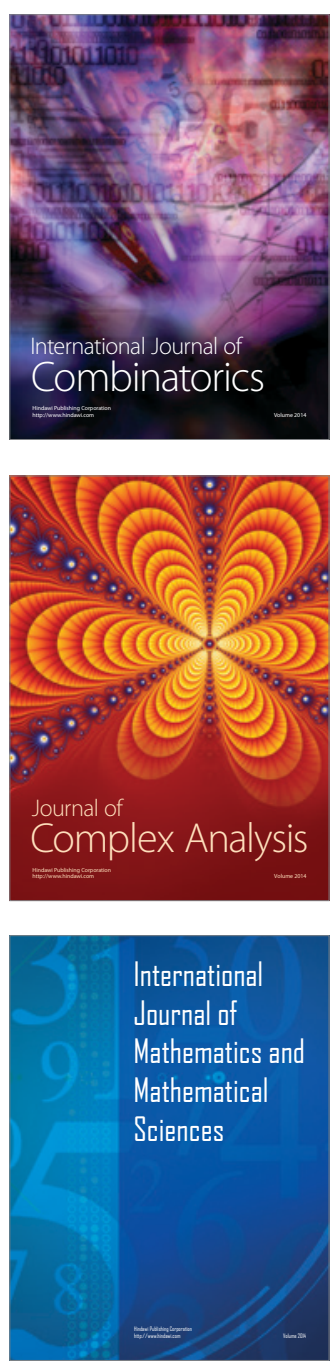
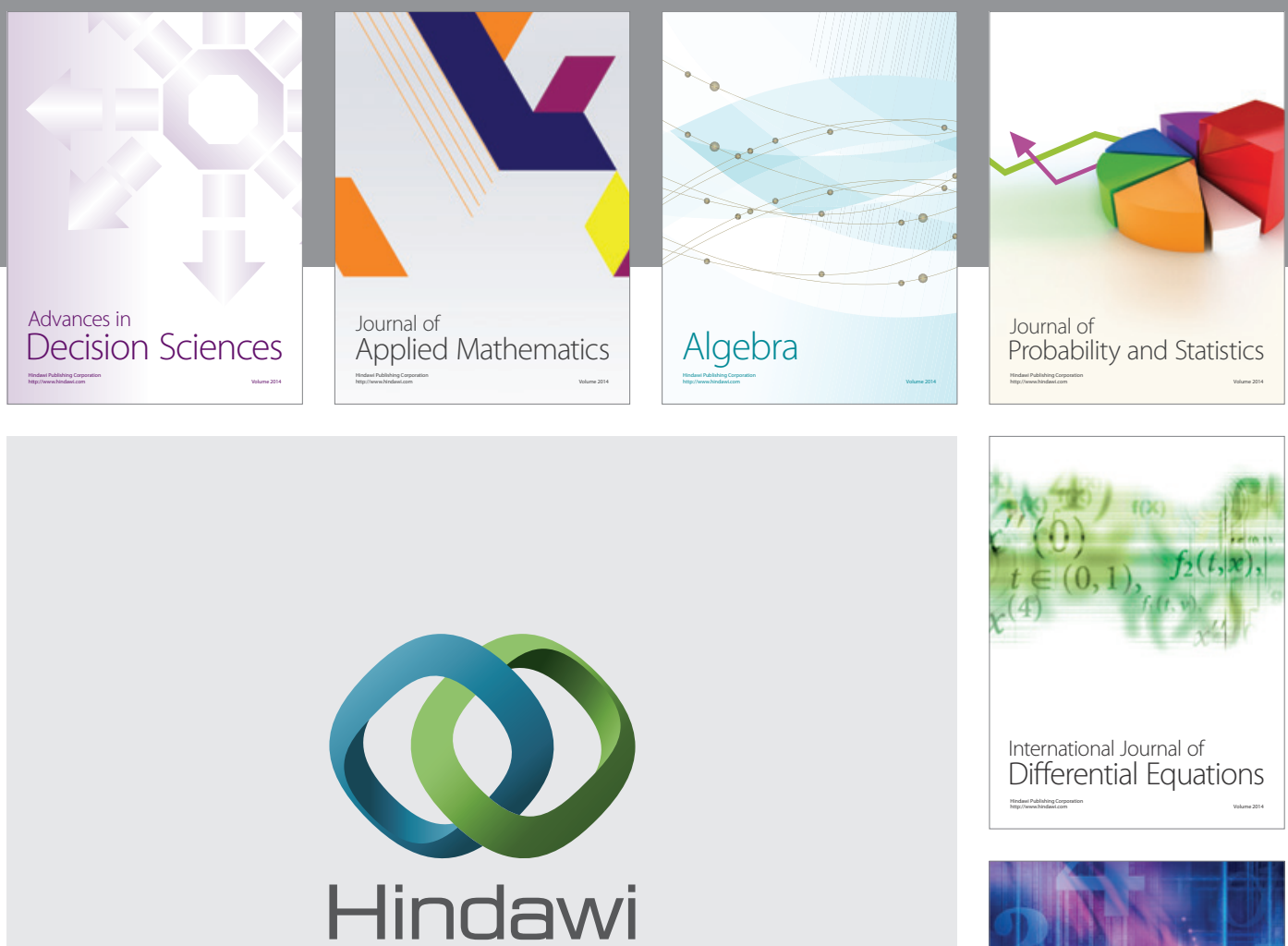

Submit your manuscripts at http://www.hindawi.com
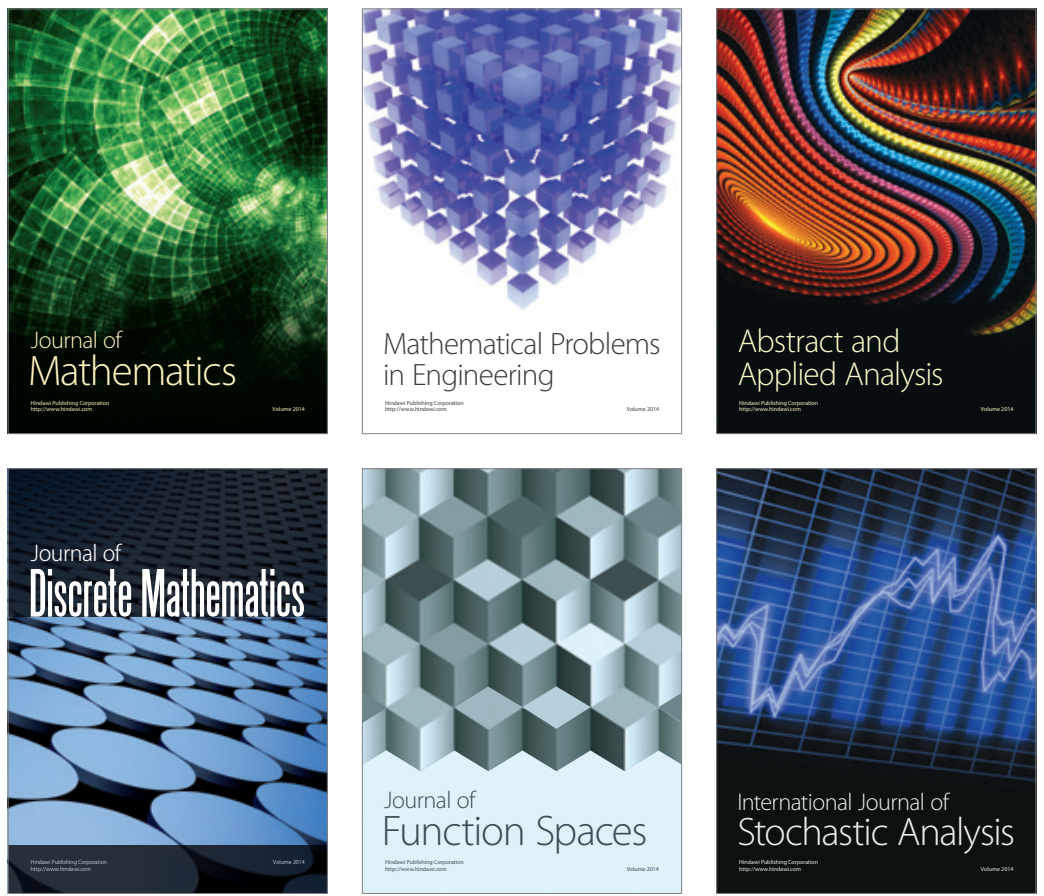

Journal of

Function Spaces

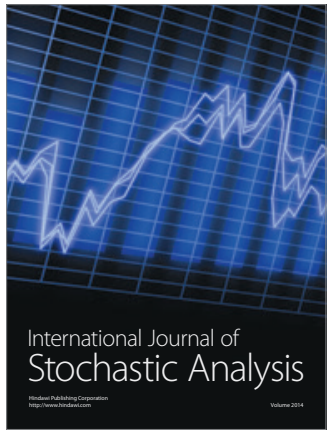

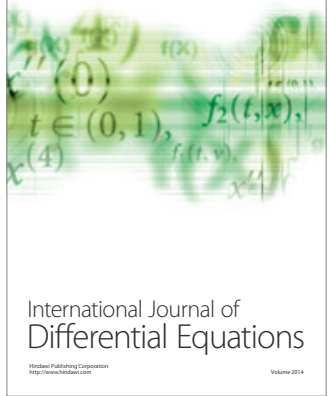
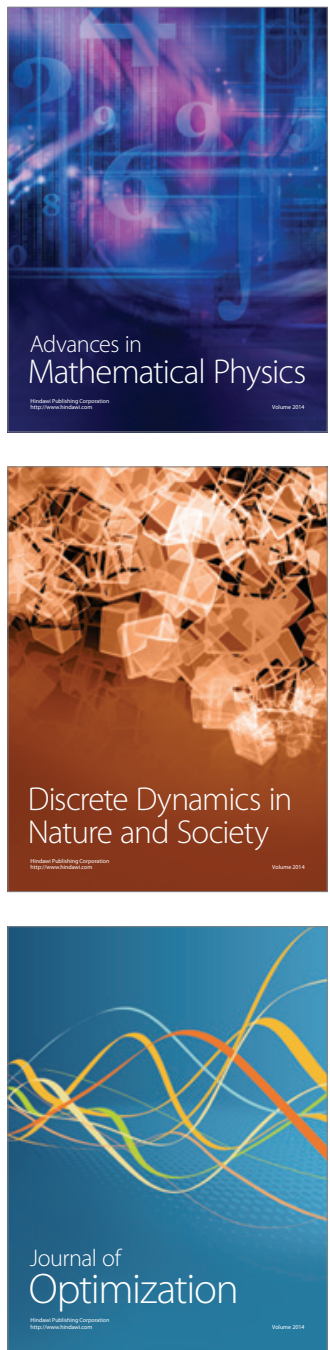